\title{
WAVELET-BASED DETECTION OF STIMULUS RESPONSES IN TIME-LAPSE MICROSCOPY
}

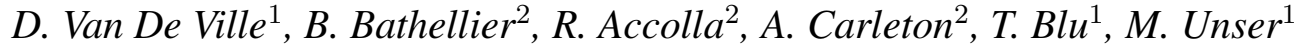 \\ ${ }^{1}$ Biomedical Imaging Group, ${ }^{2}$ Flavor Perception Group (Brain \& Mind Institute) \\ École Polytechnique Fédérale de Lausanne (EPFL) \\ CH-1015 Lausanne, Switzerland
}

\begin{abstract}
Many experimental paradigms in biology aim at studying the response to coordinated stimuli. In dynamic imaging experiments, the observed data is often not straightforward to interpret and not directly measurable in a quantitative fashion. Consequently, the data is typically preprocessed in an ad hoc fashion and the results subjected to a statistical inference at the level of a population. We propose a new framework for analyzing time-lapse images that exploits some a priori knowledge on the type of temporal response and takes advantage of the spatial correlation of the data. This is achieved by processing the data in the wavelet domain and expressing the time course of each wavelet coefficient by a linear model. We end up with a statistical map in the spatial domain for the contrast of interest (i.e., the stimulus response). The feasibility of the method is demonstrated by an example of intrinsic microscopy imaging of mice's brains during coordinated sensory stimulation.
\end{abstract}

\section{INTRODUCTION}

The study of responses to coordinated stimuli is an important topic in biomedical research. Time-lapse imaging provides spatial and temporal information that can be exploited to assess the stimulus response and its statistical significance. The proposed method, which is based on earlier work in the context of statistical analysis of functional MRI data [1], follows three main principles:

1. Spatial correlation can be efficiently exploited using the discrete wavelet transform (DWT), leading to compact representations in the wavelet domain. At the same time, the DWT is a decomposition into a basis, providing non-redundancy and perfect reconstruction. An orthogonal DWT also leaves noise evenly distributed in the wavelet domain, hence increasing the SNR of the wavelet coefficients that contribute to the signal.

2. Temporal information can be extracted using the linear model (LM) approach [2]: the correspondence with an expected stimulus response is obtained by a linear estimator.

3. The combination of the DWT and the LM for a statistical analysis resulting in a "spatial activation map".

We demonstrate the effectiveness of our method by an example of intrinsic microscopy imaging of mice's brain during coordinated sensory stimulation. The neuronal activity can be linked to a change in the refractive index which, in turn, induces variations in the reflected light. Using our approach, we are able to obtain a spatial map from a single experiment; this allows us to detect activated regions with a prescribed level of confidence (strong type-I error control). These maps can then be used for further biological interpretation.

\section{WAVELET-BASED STATISTICAL ANALYSIS}

\subsection{Spatial representation using the wavelet transform}

The DWT [3] decomposes the signal into a weighted sum of basis functions. These are shifted and dilated versions of a (bandpass) wavelet, $\psi(\mathbf{x})$, and shifted versions of a (lowpass) scaling function, $\varphi(\mathbf{x})$. For the ease of notation, we will write the 2-D spatial wavelet decomposition of a dataset $v[\mathbf{n}], \mathbf{n} \in$ $\mathbb{Z}^{2}$, as

$$
v[\mathbf{n}]=\sum_{\mathbf{k}} v_{w}[\mathbf{k}] \psi_{\mathbf{k}}(\mathbf{n}),
$$

where $\mathbf{k}$ runs over all subbands and orientations, and $\psi_{\mathbf{k}}$ is the corresponding basis function. In the context of this paper, where we have a series of images, we introduce the temporal dimension as an additional parameter $v[\mathbf{n} ; t], t=1, \ldots, N_{t}$. The practical implementation of the DWT can be done using a fast iterated filterbank algorithm.

\subsection{Temporal modelling using the linear model}

We introduce the time-course vector $\mathbf{v}_{w}[\mathbf{k}]=$ $\left[v_{w}[\mathbf{k} ; 1] \ldots v_{w}\left[\mathbf{k} ; N_{t}\right]\right]^{\mathrm{T}}$. The LM explains the temporal behavior of a wavelet coefficient with index $\mathbf{k}$ :

$$
\mathbf{v}_{w}[\mathbf{k}]=\mathbf{X y}_{w}[\mathbf{k}]+\mathbf{e}_{w}[\mathbf{k}]
$$


where the $N_{t} \times L$ design matrix $\mathbf{X}$ contains $L$ regressors, $\mathbf{y}_{w}[\mathbf{k}]$ is the parameter vector of length $L$, and $\mathbf{e}_{w}[\mathbf{k}]$ is the residual error. The design matrix should at least contain one column for the expected stimulus response and another for the constant signal. Under the hypothesis of (temporally) independently and identically gaussian-distributed residuals, the least-squares solution for the parameters is $\overline{\mathbf{y}}_{w}[\mathbf{k}]=\left(\mathbf{X}^{\mathrm{T}} \mathbf{X}\right)^{-1} \mathbf{X}^{\mathrm{T}} \mathbf{v}_{w}[\mathbf{k}]$, and the residual $\overline{\mathbf{e}}_{w}[\mathbf{k}]=$ $\mathbf{v}_{w}[\mathbf{k}]-\mathbf{X} \overline{\mathbf{y}}_{w}[\mathbf{k}]$. The contrast of interest is extracted by a so-called contrast vector $\mathbf{c}$, which results into the measures

$$
\begin{aligned}
u_{w}[\mathbf{k}] & =\mathbf{c}^{\mathrm{T}} \overline{\mathbf{y}}_{w}[\mathbf{k}], \\
s_{w}^{2}[\mathbf{k}] & =\overline{\mathbf{e}}_{w}[\mathbf{k}]^{\mathrm{T}} \overline{\mathbf{e}}_{w}[\mathbf{k}] \mathbf{c}^{\mathrm{T}}\left(\mathbf{X}^{\mathrm{T}} \mathbf{X}\right)^{-1} \mathbf{c} .
\end{aligned}
$$

The parametric approach using a LM requires coordinated stimuli and a estimation of a "typical" response derived on theoretical grounds or from emperical observation. The LM also allows to build in a certain degree of flexibility with respect to the accuracy of the stimulus response, as in [4]. For example, one can include a column with the partial derivative of the "standard" stimulus response with respect to one of its parameters (e.g., onset or decay time), orthogonalized against the "standard" response. As such, variations of the response will be captured by these additional regressors without decreasing the contrast of the standard response nor increasing the residuals.

Note that both the DWT and the LM are linear operations. Consequently, their order of execution can be interchanged and $u_{w}[\mathbf{k}]$ would corresponds to the DWT of the contrast map. However, in the next part, we introduce a powerful nonlinear treatment in the wavelet domain.

\subsection{Framework for wavelet-based statistical analysis}

\subsubsection{Wavelet denoising}

The wavelet coefficients $u_{w}[\mathbf{k}]$ are denoised in the wavelet domain based on both their estimated value and their residual error. For that purpose, we consider the proportion

$$
t_{w}[\mathbf{k}]=\frac{u_{w}[\mathbf{k}]}{\sqrt{s_{w}^{2}[\mathbf{k}] / J}}, \text { with } J=N_{t}-\operatorname{rank}(\mathbf{X}),
$$

which should follow a Student $t$-distribution in the absence of contrast (i.e., no response). The reconstruction after hard thresholding is

$\tilde{u}[\mathbf{n}]=\sum_{\mathbf{k}} \tilde{u}_{w}[\mathbf{k}] \psi_{\mathbf{k}}(\mathbf{n})$, where $\begin{cases}\tilde{u}_{w}[\mathbf{k}]=u_{w}[\mathbf{k}], & \text { when }\left|t_{w}[\mathbf{k}]\right|>\tau_{w}, \\ 0, & \text { otherwise, }\end{cases}$ where $\tau_{w}$ is a threshold parameter. Notice that we explicitly did not introduce the notion of a statistical test here.

\subsubsection{Statistical detection}

Our aim is to establish the statistical significance of the contrast in the spatial domain in order to test the hypotheses

$$
\mathcal{H}_{0}: E[\tilde{u}[\mathbf{n}]]=0, \quad \mathcal{H}_{1}: E[\tilde{u}[\mathbf{n}]]>0 .
$$

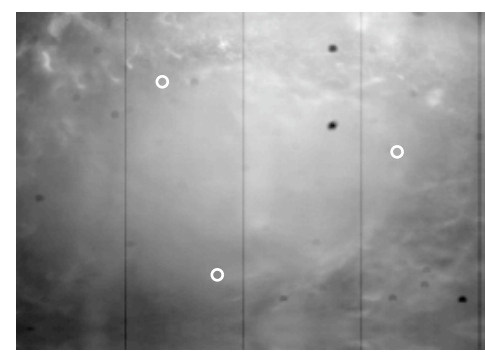

(a)

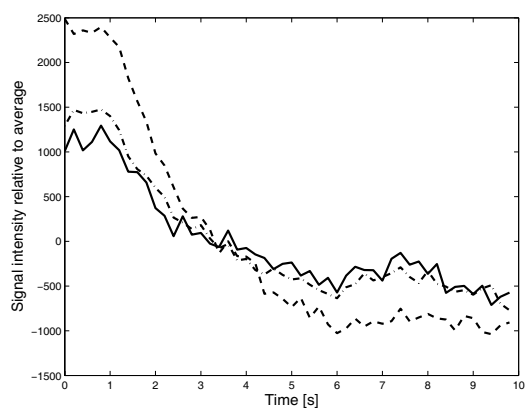

(b)

Fig. 1. (a) Typical frame of the dataset. (b) Time courses of the 3 indicated pixels with respect to their temporal average.

As a result of the theorem in [1], the test procedure relies on the null hypothesis rejection inequality:

$$
\operatorname{Prob}\left[\frac{\tilde{u}[\mathbf{n}]}{\Lambda[\mathbf{n}]} \geq \tau_{s}\right] \leq \Upsilon\left(\tau_{w}, \tau_{s}\right),
$$

where $\Upsilon\left(\tau_{w}, \tau_{s}\right)$ is data-independent, and $\Lambda[\mathbf{n}]$ is a special reconstruction of the residuals:

$$
\Lambda[\mathbf{n}]=\sum_{\mathbf{k}} \frac{s_{w}[\mathbf{k}]}{\sqrt{J}}\left|\psi_{\mathbf{k}}(\mathbf{n})\right| .
$$

In practice, the threshold values $\tau_{w}$ and $\tau_{s}$ are obtained by fixing $\Upsilon\left(\tau_{w}, \tau_{s}\right)=\alpha / N$, where $\alpha$ is the desired global significance level and $N$ the number of pixels.

\subsection{Summary of the method}

1. Apply the spatial DWT to the measured data $v[\mathbf{n} ; t]$ to obtain the wavelet coefficients $v_{w}[\mathbf{k} ; t]$.

2. Apply the LM to the temporal dimension and extract the "contrast" of interest $u_{w}[\mathbf{k}]$ with its residual error $s_{w}^{2}[\mathbf{k}]$.

3. Apply the wavelet denoising step to obtain $\tilde{u}_{w}[\mathbf{k}]$.

4. Apply the inverse DWT to $\tilde{u}_{w}[\mathbf{k}]$ and the "absolute value" inverse DWT to $s_{w}[\mathbf{k}]$. We obtain $\tilde{u}[\mathbf{n}]$ and $\Lambda[\mathbf{n}]$.

5. Apply the detection step $\tilde{u}[\mathbf{n}] / \Lambda[\mathbf{n}]>\tau_{s}$. 


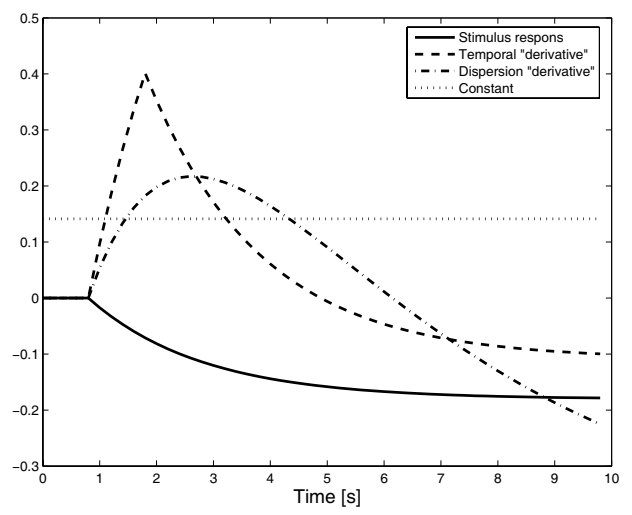

Fig. 2. Different columns of the design matrix $\mathbf{X}$, each them normalized by their energy.

\section{DYNAMIC IMAGING IN MICE'S BRAIN}

Optical imaging of brain areas is becoming increasingly important in recent developments in neurosciences; it has already revealed interesting spatial and temporal mechanisms of information coding. In this example, we consider the imaging of the brain of a mouse during coordinated sensory stimulation. The reflectance of red light is measured through an objective by a CCD camera (resolution $180 \times 252$ after binning; $5 \mathrm{~Hz}$ frame rate over 10 seconds). The stimulus-evoked intrinsic optical signal of activated brain zones is weak and noisy. Additionally, the change of the intensity is only observable with respect to the local temporal average. The aim is to reliably detect and identify the small functional units that are activated by (specific) stimuli, to allow further biological interpretation of the data. In Fig. 1 (a), we show a typical frame of the acquired dataset. In (b), the time-course of 3 highly activated pixels is plotted (with respect to their temporal average). The stimulus is applied at $t=1 \mathrm{~s}$, after which the evoked signal shows a decrease in intensity.

\subsection{Motivation}

A commonly used technique to process these data is to apply a Gaussian smoothing filter in the spatial domain, then subtract the temporal average image, and finally correlate with a typical response to the stimulus. However, Gaussian filtering brings along several problems:

- Smoothing removes potentially interesting spatial information.

- The statistical interpretation becomes complicated: while apparent SNR increased, statistical significance needs to take into account the correlation that has been introduced.
- Tuning of the width of the Gaussian filter is non-trivial: different activated zones could be of different size.

- Strong stimuli often induce a global response of the observed brain area. It is important to be able to distinguish local activation from background activation.

The multi-resolution decomposition as provided by the DWT seems to be perfectly adapted to deal with these shortcomings:

- No information is lost when taking the transform.

- The statistical interpretation is mapped into the spatial domain by using the proposed framework.

- The equivalent smoothing at each scale of the transform is adaptive and allows us to deal with the different "resolutions" of the spatial patterns present in the data.

- Wavelets automatically captures the difference of signal with respect to its local neighborhood. The global background signal resides in the lowpass subband only.

It should also be noted that the multi-resolution structure itself of the activation pattern can provide important biological insights.

\subsection{Setup}

We applied the orthogonal cubic B-spline DWT (6 iterations) to each frame of the acquisition. For the temporal modelling, the LM was setup using an empirical stimulus response derived from the observations: the stimulus response is described by a decaying exponential

$$
f\left(t ; t_{0}, t_{d}\right)= \begin{cases}e^{-\left(t-t_{0}\right) / t_{d}}-1, & t \geq t_{0}, \\ 0, & \text { otherwise },\end{cases}
$$

with constants $t_{0}$, the onset of the response, and $t_{d}$, the decay constant. Typical values are $t_{0}=1 s$ and $t_{d}=2 s$. The LM was also extended with the orthogonalized finite differences $f(t ; 2 s, 2 s)-f(t ; 1 s, 2 s)$ (to deal with delayed onsets) and the finite difference $f(t ; 1 s, 4 s)-f(t ; 1 s, 2 s)$ (to deal with variations on the decay time). The different modelling components (columns of the design matrix) are shown in Fig. 2.

\subsection{Results}

The significance level was fixed at $\alpha=0.1 \%$, which allows us to have high confidence in the detected activations since the probability of a type I error (false positive) is controlled at this level. The corresponding threshold values are $\tau_{w}=7.64$ and $\tau_{s}=0.30$. In Fig. 3 (a), we show the parameter map $u_{w}[\mathbf{k}]$ in the wavelet domain; in (b), the corresponding $t$-values $t_{w}[\mathbf{k}]$, obtained by weighting (a) with the residual error. Only $0.5 \%$ of the coefficients $u_{w}[\mathbf{k}]$ survive the thresholding operation $\left|t_{w}[\mathbf{k}]\right|>\tau_{w}$. To remove the background activation, we also 


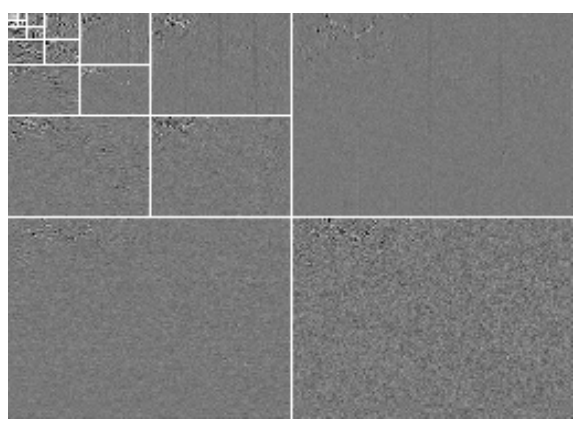

(a)

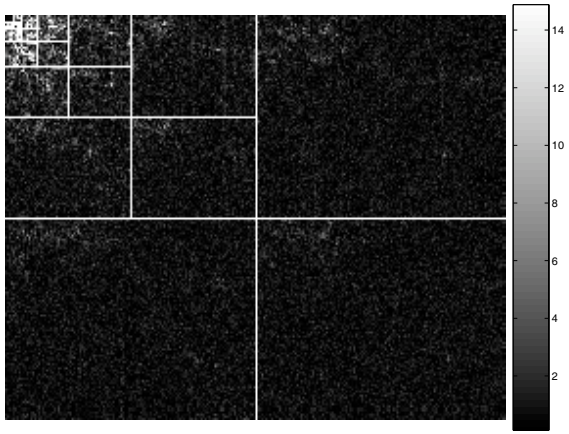

(b)

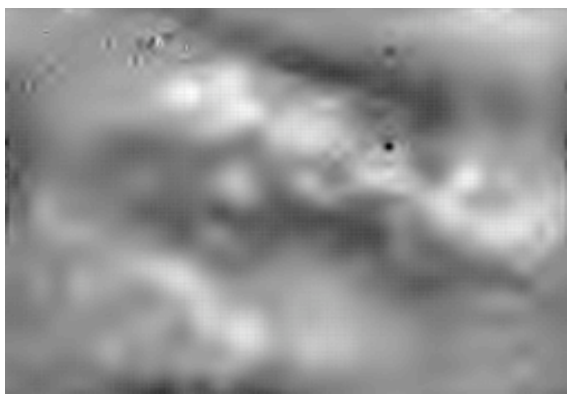

(c)

Fig. 3. (a) Wavelet coefficients $u_{w}[\mathbf{k}]$ for the stimulus respons. (b) Weighted wavelet coefficients $t_{w}[\mathbf{k}]$. (c) Significance $\tilde{u}[\mathbf{n}] / \Lambda[\mathbf{n}]$ of the stimulus response in the spatial domain.

zeroed the coefficients of the lowpass subband. Then, the contrast is reconstructed and weighted by $\Lambda[\mathbf{n}]$, shown in (c). Finally, based on the detection step $\tilde{u}[\mathbf{n}] / \Lambda[\mathbf{n}]>\tau_{s}$, we show the masked contrast in Fig. 4 (a). The statistical detection in the spatial domain selects $23 \%$ of the pixels as activated. If we reconstruct the low-pass subband only (also after tresholding), we obtain the background activation (which is statistically significant for the complete image), see Fig. 4 (b). The trade-off between the background-foreground activations is controlled by the number of iterations. These results can be used to identify a signature of the activated zones for further biological interpretation.

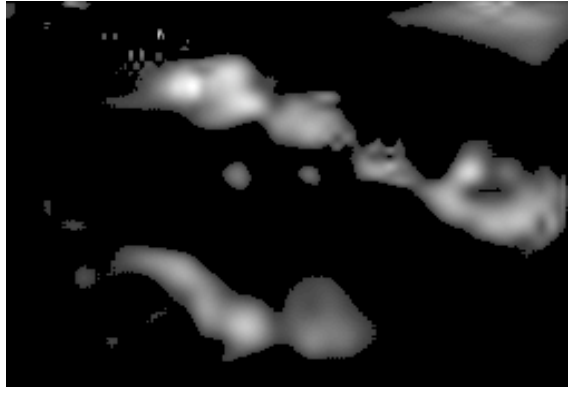

(a)

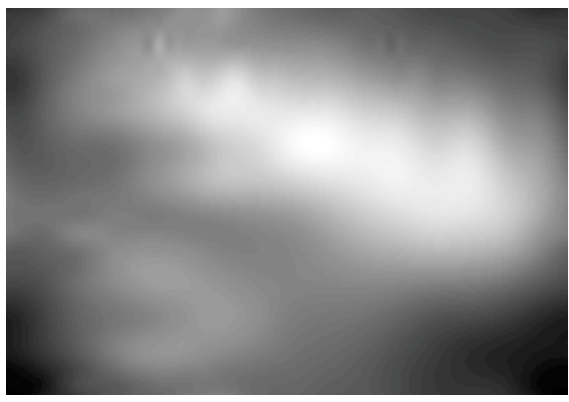

(b)

Fig. 4. (a) Stimulus response $\tilde{u}[\mathbf{n}]$ in the spatial domain masked with the detection. (b) Stimulus response of the background activation.

\section{CONCLUSION}

The proposed method allows us to obtain a statistical significance map of coordinated stimulus responses from a single experiment. The approach can be easily extended to assess multiple sessions of the same stimulus (basically by concatenation) or to study the inter-session variability (by a secondlevel analysis of the reconstructed maps $\tilde{u}[\mathbf{n}]$ ).

\section{REFERENCES}

[1] D. Van De Ville, T. Blu, and M. Unser, "Integrated wavelet processing and spatial statistical testing of fMRI data," NeuroImage, vol. 23, no. 4, pp. 1472-1485, Dec. 2004.

[2] F. A. Graybill, Theory and Application of the Linear Model, Wadsworth \& Brooks/Cole Advanced Books \& Software, Pacific Grove (CA), 1976.

[3] S. Mallat, "A theory for multiresolution signal decomposition: The wavelet decomposition," IEEE Trans. Pattern Anal. Mach. Intell., vol. 11, pp. 674-693, 1989.

[4] K. J. Friston, P. Fletcher, O. Josephs, A. Holmes, M. D. Rugg, and R. Turner, "Event-related fMRI: characterising differential response," NeuroImage, vol. 7, pp. 30-40, 1998. 